Appendix A

Measures

We used a combination of established and newly designed instruments to measure primary and secondary outcomes. These scales are described below and summarized in Table B1 (e.g., number of items, possible range, qualitative interpretation of higher scores, estimates of internal consistency reliability). Prior to conducting an impact analysis, we used pre-intervention data (i.e., step 1) from all participants to adapt and construct measures. This process involved an assessment of item-level descriptive statistics, internal consistency reliability, and the results of confirmatory factor analyses. Scales with highly skewed items or evidence of a poor fit between the data and expected factor structures were revised through item screening and follow-up exploratory factor analysis (EFA). We relied on EFA results and prior theory to guide scale construction decisions; we preferred parsimonious and theoretically-meaningful factor structures containing items loading greater than 0.40 on one factor with no loadings of this magnitude or greater on other factors.

\title{
Family Communication
}

Parent-Adolescent Communication Scale. The Parent-Adolescent Communication Scale assesses quality of communication within adolescent and caregiver dyads with a focus on openness (Barnes \& Olson, 1985). Caregivers and youth rated statements on an ordinal scale from "not at all true" to "very true."

Whole Family Communication. The Whole Family Communication scale was developed for this study based on formative qualitative work. Items assess frequency and quality of family communication activities, including problem-solving. Items measuring frequency are rated on a 6-point scale ranging from "never" to "multiple times per week". Two of the items assessing quality are rated on a 5-point scale from "never" to "very often", and the final item rates overall communication from "very unpleasant" to "very pleasant". Items were standardized, summed to create a scale, and the resulting scale was standardized to have a mean of 0 and standard deviation of 1.

Frequency of Communication about Sex and HIV. The Frequency of Communication about Sex and HIV scale includes items adapted from two existing scales of 
parent-child communication related to sexual risk (Miller et al., 1998; Hutchinson et al., 2003). Both caregivers and youth completed this measure, responding to statements on a 4-point scale describing the frequency with which they discuss topics related to sex and sexual risk.

Quality of Communication About Sex. The Quality of Communication About Sex scale was developed by Miller et al. (Miller et al., 1998) and includes items related to the helpfulness and openness of caregiver-child communication about sex. Both caregivers and youth completed the measure, and responses include a 4-point likert scale from "strongly disagree" to "strongly agree".

Economic Support Communication. The Economic Support Communication scale was developed for this study from qualitative formative work and assesses communication regarding the provision of basic needs from the child's perspective. Items focused on discussions about the child's needs and reasons they can or cannot be met, as well as the child's perceptions of the degree to which caregivers' are concerned about providing for them. Youth responded on 4-point likert scales reflecting magnitude or frequency.

\section{Parenting and Social Support}

Alabama Parenting Questionnaire. Parenting practices were assessed with a subset of items from the Alabama Parenting Questionnaire (APQ) (Shelton et al., 1996). Confirmatory factor analysis revealed a poor of the data to the original 6-factor model. Results of an exploratory factor analysis suggested a 3-factor model reflecting parental involvement, positive parenting, and negative parenting. Both caregivers and youth completed this measure, responding on a 5-point likert scale ranging from "never" to "always". Youth reported about joint behavior of both caregivers, if more than one, on positive and negative parenting subscales, but about caregivers separately on the involvement subscale.

Network of Relationships Inventory. The Network of Relationships Inventory (Furman \& Buhrmester, 1985) is a 45-item measure of social support. For this study, we assessed caregiver-provided support and calculated two composite scores: Social Support and Negative Interactions. Only youth completed this measure, responding on a 5-point scale ranging from "Little or none", to "The most". 


\section{HIV Risk Indicators}

Brief HIV Knowledge Questionnaire. The Brief HIV Knowledge Questionnaire (Carey \& Schroder, 2002) measures the respondent's knowledge about HIV with a focus on etiology and transmission. Response choices were yes, no, or don't know.

Sex Self-Efficacy. Five items were used to assess youths' self-efficacy related to protective sexual behavior, including condom use and refusing unwanted sex. Three items were drawn from the Self-Efficacy to Refuse Sexual Behavior Scale (Cecil \& Pinkerton, 1998), and two were developed for this study. Youth reported their level of confidence on a scale from 1 to 5 .

Sex Beliefs. The Sex Beliefs scale is comprised of items that measure endorsement of beliefs associated with sexual risk (e.g., "It is ok for men to have multiple partners."). Youth responded to statements on a 4-point scale indicating level of agreement.

\section{Mental Health}

To assess mental health, we administered three established measures widely used in Western contexts, including the Multi-Dimensional Anxiety Scale for Children 10-item short version (March et al., 1997); the Children's Depression Inventory (Kovacs, 1992); the Strengths and Difficulties Questionnaire (Goodman, 2001). Confirmatory factor analyses and item-level descriptives revealed problems with several items, leading us to use a reduced number of well-performing items. 
Appendix B

Supplementary Figures and Tables 


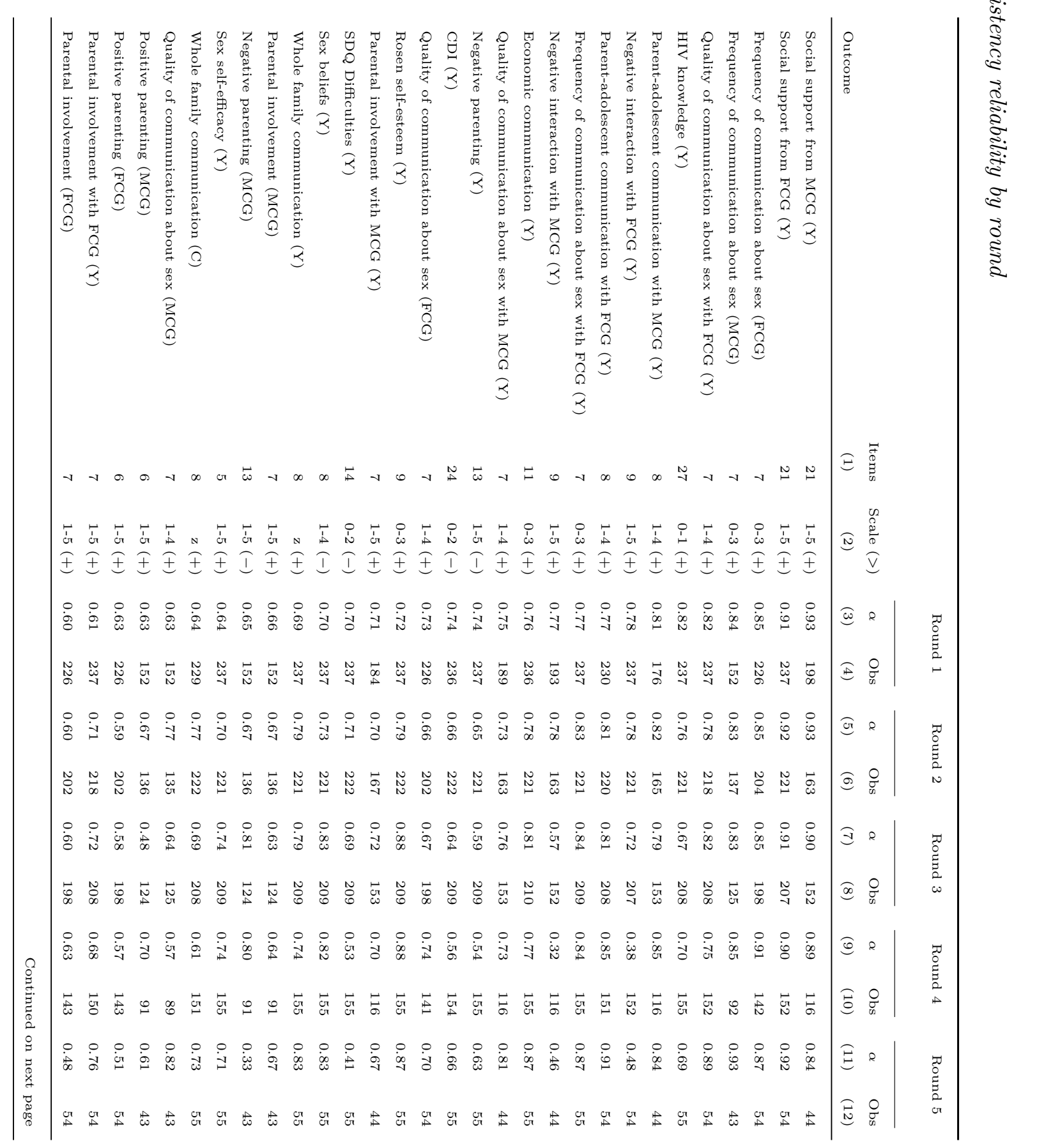




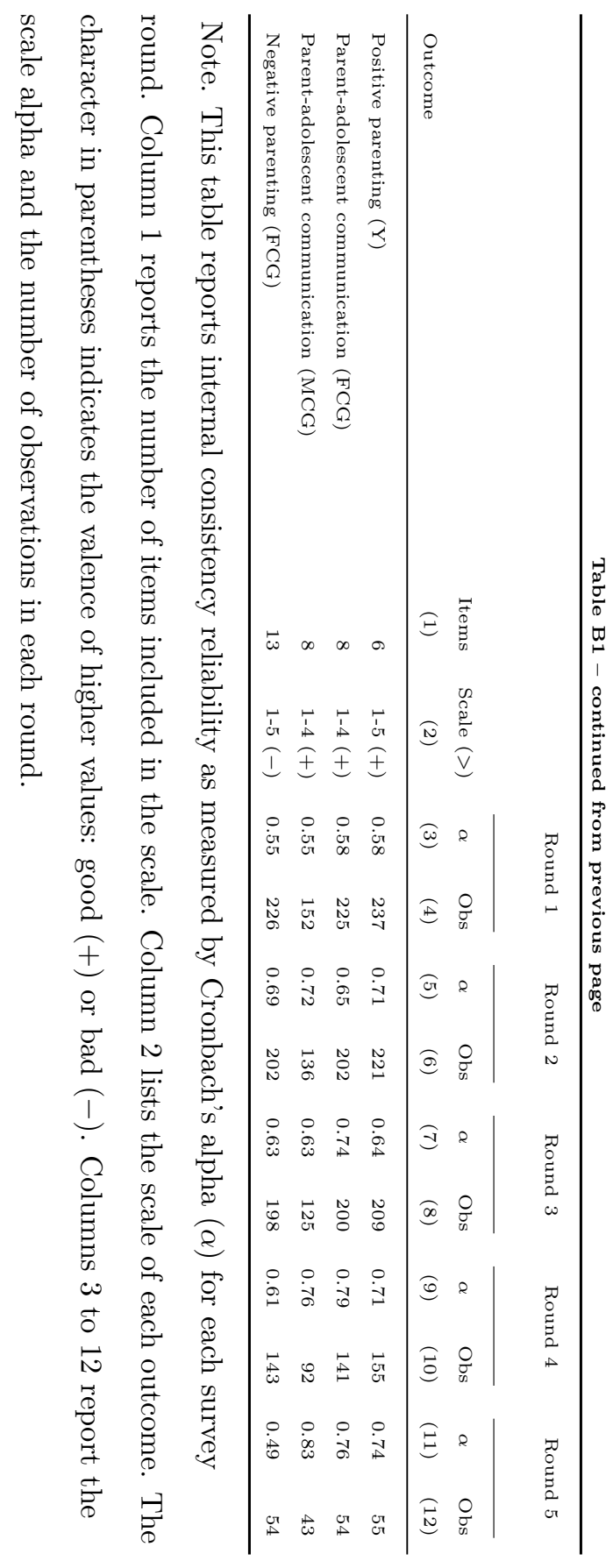


Table B2

Treatment compliance

\begin{tabular}{|c|c|c|c|c|c|c|c|c|c|c|}
\hline \multirow[b]{2}{*}{ Participants } & \multicolumn{2}{|c|}{ All } & \multicolumn{2}{|c|}{ Church 1} & \multicolumn{2}{|c|}{ Church 2} & \multicolumn{2}{|c|}{ Church 3} & \multicolumn{2}{|c|}{ Church 4} \\
\hline & $N$ & Value & $n$ & Value & $n$ & Value & $n$ & Value & $n$ & Value \\
\hline Family treatment compliance $(\%)$ & 125 & 75.2 & 34 & 67.6 & 28 & 67.9 & 33 & 81.8 & 30 & 83.3 \\
\hline Male caregiver attendance rate & 81 & 41.7 & 20 & 27.8 & 17 & 51.0 & 20 & 36.7 & 24 & 50.9 \\
\hline Female caregiver attendance rate & 120 & 61.8 & 34 & 59.8 & 27 & 65.4 & 32 & 59.4 & 27 & 63.4 \\
\hline Couples attendance rate & 77 & 28.7 & 20 & 25.6 & 16 & 36.8 & 20 & 21.7 & 21 & 32.3 \\
\hline Youth attendance rate & 237 & 72.6 & 67 & 75.6 & 41 & 69.9 & 69 & 69.2 & 60 & 74.8 \\
\hline
\end{tabular}

Note. "Family treatment compliance" is defined as at least one caregiver and at least one youth attending 5 or more sessions of the 9 session intervention; the value represents the percentage of compliant families (households). Attendance rates are calculated as the number of sessions attended divided by 9, the maximum possible sessions; the value represents the mean attendance rate. "Couples attendance rate" is the mean proportion of sessions attended by the female caregiver and male caregiver among 2-caregiver homes. 
Table B3

Comparison of baseline $(j=1)$ characteristics among found and unfound youth

\begin{tabular}{|c|c|c|c|c|c|}
\hline \multirow[b]{2}{*}{ Variable } & \multicolumn{2}{|c|}{$\begin{array}{l}\text { Found } \\
(n=215)\end{array}$} & \multicolumn{2}{|c|}{$\begin{array}{l}\text { Unfound } \\
(n=22)\end{array}$} & \multirow[b]{2}{*}{ p-value } \\
\hline & Mean & $\mathrm{SD}$ & Mean & $\mathrm{SD}$ & \\
\hline Age & 12.22 & 1.99 & 12.82 & 2.40 & 0.193 \\
\hline Female & 0.50 & 0.50 & 0.68 & 0.48 & 0.109 \\
\hline Attends school & 0.99 & 0.12 & 1.00 & 0.00 & 0.579 \\
\hline Orphan & 0.34 & 0.48 & 0.55 & 0.51 & 0.062 \\
\hline Luo tribe & 0.60 & 0.49 & 0.68 & 0.48 & 0.432 \\
\hline Negative parenting (FCG) & 1.50 & 0.38 & 1.36 & 0.24 & 0.103 \\
\hline Parental involvement (FCG) & 3.33 & 0.67 & 3.44 & 0.78 & 0.485 \\
\hline Positive parenting (FCG) & 3.68 & 0.63 & 3.42 & 0.73 & 0.065 \\
\hline Frequency of communication about sex (FCG) & 1.05 & 0.93 & 1.05 & 0.68 & 0.983 \\
\hline Frequency of communication about sex (MCG) & 1.02 & 0.85 & 0.87 & 0.61 & 0.576 \\
\hline Negative parenting (MCG) & 1.60 & 0.42 & 1.73 & 0.59 & 0.356 \\
\hline Parental involvement (MCG) & 3.35 & 0.69 & 3.65 & 0.51 & 0.160 \\
\hline Positive parenting (MCG) & 3.60 & 0.63 & 3.88 & 0.30 & 0.149 \\
\hline Parent-adolescent communication (FCG) & 3.03 & 0.43 & 2.99 & 0.38 & 0.722 \\
\hline Parent-adolescent communication (MCG) & 3.00 & 0.38 & 3.06 & 0.34 & 0.641 \\
\hline Quality of communication about sex (FCG) & 2.89 & 0.56 & 2.66 & 0.66 & 0.075 \\
\hline Quality of communication about sex (MCG) & 2.85 & 0.47 & 2.69 & 0.44 & 0.284 \\
\hline SDQ Difficulties (C) & 0.65 & 0.34 & 0.73 & 0.31 & 0.289 \\
\hline SDQ Strengths (C) & 1.72 & 0.28 & 1.83 & 0.22 & 0.080 \\
\hline Negative parenting $(Y)$ & 1.77 & 0.46 & 1.52 & 0.46 & 0.014 \\
\hline Positive parenting (Y) & 3.27 & 0.57 & 3.52 & 0.75 & 0.054 \\
\hline
\end{tabular}


Table B3 - continued from previous page

\begin{tabular}{|c|c|c|c|c|c|}
\hline \multirow[b]{2}{*}{ Variable } & \multicolumn{2}{|c|}{$\begin{array}{l}\text { Found } \\
(n=215)\end{array}$} & \multicolumn{2}{|c|}{$\begin{array}{l}\text { Unfound } \\
(n=22)\end{array}$} & \multirow[b]{2}{*}{ p-value } \\
\hline & Mean & $\mathrm{SD}$ & Mean & $\mathrm{SD}$ & \\
\hline CDI $(Y)$ & 0.27 & 0.20 & 0.20 & 0.13 & 0.105 \\
\hline Church support, ask leader (Y) & 0.45 & 0.77 & 0.71 & 1.14 & 0.147 \\
\hline Church support, ask member (Y) & 0.75 & 1.05 & 1.12 & 1.44 & 0.145 \\
\hline Coping, active $(Y)$ & 0.63 & 0.27 & 0.62 & 0.25 & 0.958 \\
\hline Coping, avoidant $(\mathrm{Y})$ & 0.55 & 0.26 & 0.68 & 0.29 & 0.035 \\
\hline Economic communication (Y) & 1.69 & 0.55 & 1.63 & 0.67 & 0.627 \\
\hline Whole family communication (Y) & 2.67 & 0.82 & 2.97 & 1.06 & 0.115 \\
\hline Parental involvement with FCG (Y) & 2.76 & 0.70 & 3.06 & 0.81 & 0.065 \\
\hline Social support from FCG (Y) & 3.56 & 0.77 & 3.81 & 0.62 & 0.153 \\
\hline Negative interaction with FCG (Y) & 4.43 & 0.53 & 4.72 & 0.26 & 0.013 \\
\hline Quality of communication about sex with FCG (Y) & 2.51 & 0.64 & 2.52 & 0.73 & 0.899 \\
\hline Frequency of communication about sex with FCG (Y) & 0.62 & 0.66 & 0.58 & 0.78 & 0.756 \\
\hline HIV knowledge (Y) & 0.68 & 0.17 & 0.65 & 0.29 & 0.495 \\
\hline Parental involvement with MCG (Y) & 2.53 & 0.79 & 2.51 & 0.88 & 0.931 \\
\hline Social support from MCG (Y) & 2.96 & 0.92 & 2.96 & 0.96 & 0.992 \\
\hline Negative interaction with MCG (Y) & 4.53 & 0.48 & 4.80 & 0.31 & 0.031 \\
\hline Quality of communication about sex with MCG (Y) & 2.41 & 0.59 & 2.22 & 0.65 & 0.226 \\
\hline Parent-adolescent communication with MCG (Y) & 2.90 & 0.53 & 2.73 & 0.63 & 0.260 \\
\hline Parent-adolescent communication with FCG (Y) & 3.12 & 0.46 & 3.24 & 0.39 & 0.246 \\
\hline Religious coping (Y) & 2.50 & 0.41 & 2.67 & 0.32 & 0.069 \\
\hline Rosen self-esteem (Y) & 2.14 & 0.40 & 2.20 & 0.29 & 0.507 \\
\hline SDQ Difficulties (Y) & 0.52 & 0.32 & 0.57 & 0.29 & 0.443 \\
\hline SDQ Strengths (Y) & 1.75 & 0.30 & 1.88 & 0.19 & 0.038 \\
\hline
\end{tabular}


Table B3 - continued from previous page

\begin{tabular}{|c|c|c|c|c|c|}
\hline \multirow[b]{2}{*}{ Variable } & \multicolumn{2}{|c|}{$\begin{array}{l}\text { Found } \\
(n=215)\end{array}$} & \multicolumn{2}{|c|}{$\begin{array}{l}\text { Unfound } \\
(n=22)\end{array}$} & \multirow[b]{2}{*}{$\mathrm{p}$-value } \\
\hline & Mean & $\mathrm{SD}$ & Mean & $\mathrm{SD}$ & \\
\hline Sex self-efficacy $(Y)$ & 2.93 & 1.00 & 3.22 & 1.09 & 0.204 \\
\hline Sex beliefs $(Y)$ & 1.72 & 0.44 & 1.61 & 0.42 & 0.270 \\
\hline Stress events $(\mathrm{Y})$ & 0.47 & 0.20 & 0.52 & 0.19 & 0.237 \\
\hline Traumatic events (Y) & 0.41 & 0.28 & 0.44 & 0.39 & 0.683 \\
\hline Trauma symptoms (Y) & 1.33 & 0.66 & 1.21 & 0.65 & 0.431 \\
\hline Ever vaginal sex & 0.24 & 0.43 & 0.36 & 0.49 & 0.193 \\
\hline High risk sex & 0.87 & 0.34 & 0.60 & 0.55 & 0.123 \\
\hline
\end{tabular}

Note. Attrition defined as being unfound at the first post-intervention observation: $j=2$ for youth from

Church $1, j=3$ for youth from Church $2 ; j=4$ for youth from Church $3 ; j=5$ for youth from Church 4 .

Abbreviations: $\mathrm{Y}=$ Youth $\mathrm{C}=$ Caregiver $\mathrm{MCG}=$ Male caregiver; $\mathrm{FCG}=$ Female caregiver. 